Revue d'histoire de l'Amérique française

MA REVUE D.HISTOIRE DE L'AMÉRIQUE FRANÇAISE

\title{
Histoire sociale et démographique d'une communauté isolée : Saint-Barthélemy (Antilles françaises)
}

\section{Yolande Lavoie}

Volume 42, numéro 3, hiver 1989

URI : https://id.erudit.org/iderudit/304708ar

DOI : https://doi.org/10.7202/304708ar

Aller au sommaire du numéro

Éditeur(s)

Institut d'histoire de l'Amérique française

ISSN

0035-2357 (imprimé)

1492-1383 (numérique)

Découvrir la revue

Citer cette note

Lavoie, Y. (1989). Histoire sociale et démographique d'une communauté isolée : Saint-Barthélemy (Antilles françaises). Revue d'histoire de l'Amérique française, 42(3), 411-427. https://doi.org/10.7202/304708ar d'utilisation que vous pouvez consulter en ligne. 
NOTE DE RECHERCHE

\section{HISTOIRE SOCIALE ET DÉMOGRAPHIQUE D'UNE COMMUNAUTÉ ISOLÉE: SAINT-BARTHÉLEMY (Antilles françaises)}

YOLANDE LAVOIE

Département de démographie

Université de Montréal

Depuis quelques décennies, de nombreuses équipes et des chercheurs isolés s'attachent à reconstruire des populations sur plusieurs siècles, individu par individu. Grâce aux liens conjugaux et de filiation, il leur est possible de reconstituer les familles et les généalogies et d'étudier le renouvellement des populations choisies. Les approches longitudinale et intergénérationnelle qui sous-tendent ce type de recherches, malgré leurs incontestables avantages, sont peu adaptées à la détection des événements conjoncturels (crises, guerres, épidémies) et à l'analyse de leurs répercussions (mécanismes régulateurs et retour à l'équilibre). Pourtant le destin des individus est marqué non seulement par l'appartenance à une cohorte donnée, mais aussi par la conjoncture propre à leur époque. La trame événementielle, assortie de données statistiques, de témoignages et d'indications des comportements démographiques, est d'une inestimable valeur pour interpréter les données relatives à la vie sociale et au renouvellement des populations.

Notre équipe ${ }^{1}$ exploite et enrichit depuis plusieurs années le registre de population de l'île Saint-Barthélemy (Antilles françaises) aux fins d'études génétiques et démographiques. En cela, elle partage pleinement les objectifs de SOREP, Centre interuniversitaire de recherches sur les populations de Chicoutimi et, au plan démographique, ceux du PRDH (Programme de recherche en démographie historique) de Mont-

\footnotetext{
1 Le présent texte a été rédigé dans le cadre du Programme de l'Équipe de recherches multidisciplinaires sur la dynamique des populations humaines (EDYPH). Ce programme a pour but de mettre en lumière, à partir de registres de population constitués en bases de données, les facteurs biologiques et sociaux qui contribuent au renouvellement des populations étudiées. L'île Saint-Barthélemy est l'un des milieux pris en compte par le programme d'EDYPH. Cette recherche a été rendue possible grâce aux subventions du Conseil de recherches en sciences humaines du Canada.
} 
réal. Alors que le centre SOREP, qui concentre particulièrement ses activités sur la région saguenayenne, a été progressivement amené à une perspective multirégionale englobant Charlevoix et le Bas SaintLaurent, le PRDH, d'emblée, choisissait de reconstituer l'entière population du Québec ancien. Notre registre est de taille beaucoup plus modeste puisque l'île Saint-Barthélemy, population et territoire, se compare assez bien à l'Ile-aux-Coudres (Québec) ${ }^{2}$. Saint-Barthélemy n'est donc qu'une infime parcelle de la Franco-Amérique qui aurait pu, sans la pratique prolongée de l'esclavage et l'expérience d'un siècle de domination suédoise, s'apparenter de façon encore plus étroite à l'Ileaux-Coudres puisque les deux populations insulaires trouvent leur origine dans les tentatives de colonisation qui ont permis à la France de s'implanter au XVIIe siècle dans l'Amérique septentrionale et dans les Antilles.

Les travaux du PRDH trouvent naturellement un cadre historique très riche dans les nombreux ouvrages qui portent sur la NouvelleFrance et le Québec ancien ${ }^{3}$. Moins favorisé à cet égard, le centre SOREP a entrepris d'établir ce cadre dans un ouvrage considérable dont les analyses s'appuient sur une abondante documentation ${ }^{4}$. En quête également d'une image organisée de l'évolution de la population de SaintBarthélemy, nous avons ramené nos ambitions à des proportions plus compatibles avec l'importance géographique et démographique de l'île.

À partir d'oeuvres d'historiens, de géographes, de généalogistes et également de documents d'archives, nous avons donc tenté de dégager la trame sur laquelle s'élaborent les recherches en cours, soit, d'une part, la constitution du noyau de quelques dizaines de personnes dont les lignées forment encore aujourd'hui l'armature de l'île et, d'autre part, le durcissement de ce noyau en dépit des risques d'éclatement ou d'absorption auxquels l'histoire l'a exposé.

Les circonstances historiques, les clivages sociaux, les migrations externes parfois massives et un vigoureux accroissement naturel ont, simultanément ou en alternance, contribué à modeler le milieu insulaire. L'impact sur l'effectif démographique d'une histoire heurtée et mouvementée n'est pas négligé dans ce texte. Toutefois, c'est principalement sur les facteurs qui ont concouru parfois à infléchir l'évolution démographique mais, le plus souvent, à y introduire des ruptures notables que nous mettons l'accent.

\footnotetext{
$2 \quad$ L'île-aux-Coudres est comprise dans le programme de recherches de l'EDYPH; sa population a été étudié par Pierre Philippe, dans Étude des effets de la consanguinité sur quelques facteurs de la fécondité. Thèse de doctorat (anthropologie), Université de Montréal, 1972.

3 Hubert Charbonneau, La population du Québec: études rétrospectives (Montréal, Les Éditions du Boréal Express, 1973), 5-22.

4 Christian Pouyez et Yolande Lavoie, Les Saguenayens. Introduction à l' histoire des populations du Saguenay XVIe-XXe siècles (Québec, Presses de l'Université du Québec, 1983), 386 p.
} 


\section{FLUCTUATIONS DÉMOGRAPHIQUES ET TRAME HISTORIQUE}

L'histoire de Saint-Barthélemy explique en grande partie son évolution démographique. Le peuplement de l'île a subi les contrecoups des heurts entre la France et l'Angleterre aux XVIIe et XVIIIe siècles. C'est au début du XVIIe siècle que la France, préoccupée d'imposer sa présence dans les îles d'Amérique, à l'instar de l'Angleterre et de la Hollande, cherche à installer des colons dans les Antilles mineures, négligées par l'Espagne qui s'était accaparé les grandes Antilles. Après un premier essai de colonisation (1648), auquel met fin le massacre des habitants par les indiens caraïbes (1656), une trentaine de colons français, en provenance de Saint-Christophe, s'installent en 1659 à SaintBarthélemy, accompagnés de quelques esclaves. Leur nombre devait atteindre la centaine en 1664, selon le Père Dutertre. En 1666, les habitants de Saint-Barthélemy sont appelés à occuper les terres de la partie anglaise de Saint-Christophe reprise par les Français. On leur substitue de 700 à 800 Irlandais évacués de la partie conquise de Saint-Christophe. Cet échange n'a pas eu de suite: une partie au moins des colons reviennent à Saint-Barthélemy que les Irlandais doivent abandonner. La guerre de la Ligue d'Augsbourg (1688-1697) a des répercussions dans les Antilles et le développement de Saint-Barthélemy s'en trouve freiné ${ }^{5}$. Jusqu'au traité d'Utrecht (1713), la France se montre réticente au repeuplement de l'île, de sorte que, vers 1730, la population blanche s'élève à tout au plus 200 habitants.

Les guerres de la Succession d'Autriche (1744-1756) et de Sept Ans (1756-1763) touchent à leur tour Saint-Barthélemy. La population, évacuée en 1747 par les corsaires anglais, ne parvient pas à se réimplanter dans l'île avant 1763 (tableau 1 et figure 1). Les avantages stratégiques de l'île perdant de l'intérêt, la France la cède en 1784 à la Suède contre un droit d'entrepôt à Göteborg. Au moment de cette cession, l'île compte 739 habitants. La vigueur de l'activité économique suscitée par les Suédois portera la population à environ 6000 habitants en 1815. C'est l'apogée. En trente ans, la population rurale de souche a plus que doublé, mais la croissance est surtout due à la création de Gustavia, ville portuaire, qui a attiré dans l'île des milliers d'étrangers ${ }^{6}$. Entre 1820 et 1840 , l'effectif de la population rurale régresse de plus d'une centaine d'individus, alors qu'à Gustavia, desservie par le déclin rapide du commerce, on ne dénombre, en 1840, qu'un millier d'habitants (tableau 1). La Suède, pour laquelle l'île est devense un fardeau, cherche à y intéresser d'autres puissances. Finalement, elle la rétrocède à la France en 1878.

\footnotetext{
6 Georges Bourdin, Histoire de Saint-Barthélemy, (Pelhams, H. Porter, 1978), 112-114. 27.
} 


\section{TABLEAU 1}

Évolution des effectifs de la population de Saint-Barthélemy selon l'habitat (1664-1981)

\begin{tabular}{|c|c|c|c|c|c|c|c|c|c|}
\hline \multirow[b]{2}{*}{ Année } & \multicolumn{4}{|c|}{ Campagne } & \multicolumn{4}{|c|}{ Ville } & \multirow{2}{*}{ 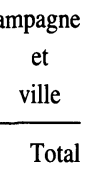 } \\
\hline & Total & Blancs & Libres & Escl. & Total & Blancs & Libres & Escl. & \\
\hline 1664 & 100 & & & & & & & & \\
\hline 1671 & 336 & 290 & & 46 & & & & & \\
\hline 1687 & 501 & 355 & & 141 & & & & & \\
\hline 1700 & 90 & 85 & & 5 & & & & & \\
\hline 1731 & 347 & 209 & & 138 & & & & & \\
\hline 1747 & 710 & 310 & & 400 & & & & & \\
\hline 1756 & 0 & & & & & & & & \\
\hline 1765 & 371 & 258 & & 113 & & & & & \\
\hline 1767 & 579 & 328 & & $251^{*}$ & & & & & \\
\hline 1775 & 754 & 419 & & $335^{*}$ & & & & & \\
\hline 1784 & 739 & 458 & & $281^{*}$ & & & & & \\
\hline 1788 & 978 & 543 & & 435 & 583 & 311 & & 272 & 1561 \\
\hline 1794 & 1068 & 547 & & 521 & 1144 & 590 & & 554 & 2212 \\
\hline 1812 & 1601 & 933 & 90 & 588 & 3881 & 1025 & 1308 & 1818 & 5482 \\
\hline 1819 & 1677 & & & & 2910 & & & & 4587 \\
\hline 1834 & 1640 & & & & 2080 & & & & 3720 \\
\hline 1838 & 1553 & & & 358 & 1412 & & & 338 & 2965 \\
\hline 1840 & 1498 & 1049 & 69 & 380 & 1057 & & & & 2555 \\
\hline 1846 & 1499 & 1022 & 142 & 335 & 1131 & 290 & 641 & 200 & 2649 \\
\hline 1854 & 1683 & 1225 & 458 & & 1148 & 304 & 839 & & 2831 \\
\hline 1866 & 1990 & & & & 908 & & & & 2898 \\
\hline 1872 & 1549 & & & & 841 & & & & 2390 \\
\hline 1875 & 1581 & & & & 793 & & & & 2374 \\
\hline 1897 & 2003 & & & & 790 & & & & 2793 \\
\hline 1921 & & & & & & & & & 2519 \\
\hline 1935 & & & & & & & & & 2734 \\
\hline 1946 & 1839 & & & & 935 & & & & 2234 \\
\hline 1954 & 1757 & & & & 322 & & & & 2079 \\
\hline 1961 & 1892 & & & & 324 & & & & 2216 \\
\hline 1967 & & & & & & & & & 2350 \\
\hline 1974 & & & & & & & & & 2521 \\
\hline 1981 & & & & & & & & & 3500 \\
\hline
\end{tabular}

* Comprend peut-être quelques personnes de couleur libres.

Sources: EDYPH (à partir de nombreux documents et citations).

\section{L'ÉVOLUTION PATRONYMIQUE: INDICE DE L'ÉMERGENCE DU NOYAU FRANÇAIS}

De telles données historiques et numériques suggèrent d'importants changements dans les éléments constitutifs de la population. On peut, pour illustrer l'ampleur des variations de la composition de la population rurale, renvoyer à celles de la distribution patronymique. 
FIGURE 1

Évolution de la population de l'île Saint-Barthélemy selon l'habitat, 1664-1981

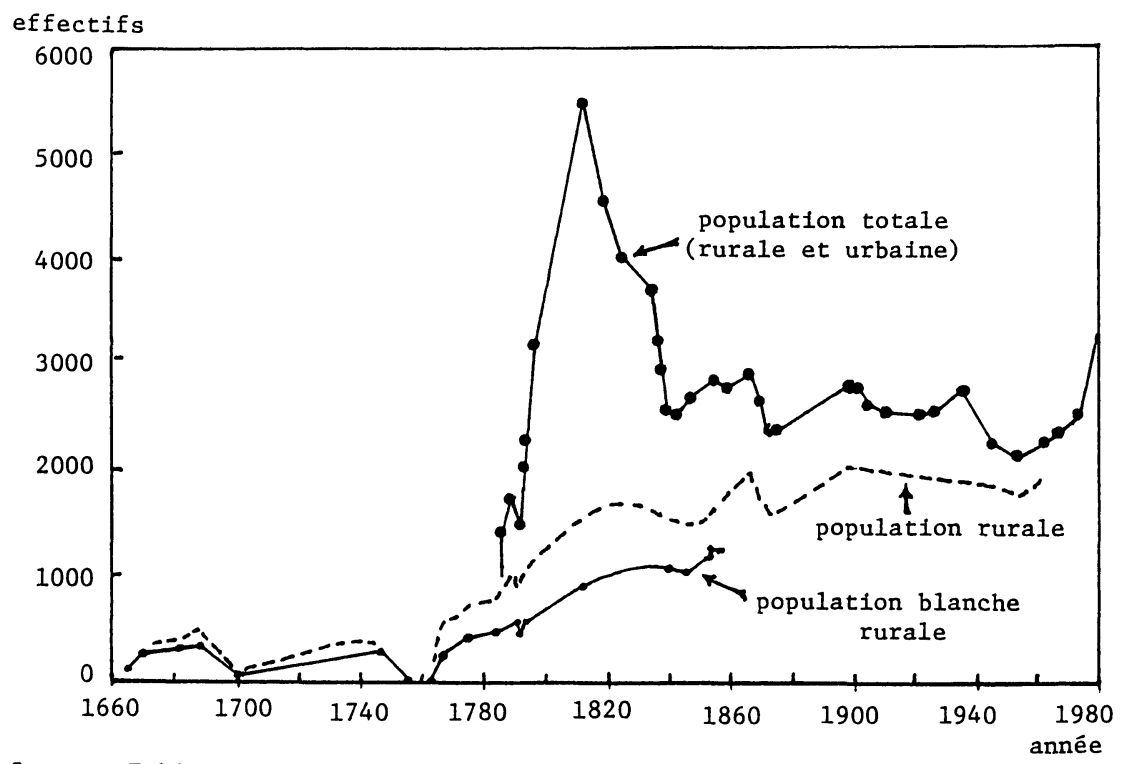

Source: Tableau 1

Depuis longtemps, les noms de famille ont constitué un matériau statistique très riche, utilisé notamment par les historiens, les démographes, les ethnologues et les généticiens. L'évolution des patronymes dans une société donnée peut éclairer la transmission génétique. Elle peut aussi révéler les grands traits de la dynamique de la population, c'est-à-dire l'ensemble des traits non biologiques qui caractérisent le destin d'un groupe humain sur un territoire. ${ }^{7}$

Plusieurs recensements et listes nous permettent de suivre l'évolution du stock des patronymes des débuts du peuplement jusqu'à aujourd'hui. En 1681, le «Rolle des habitans» porte 295 blancs, répartis en 73 ménages dont deux dirigés par des veuves ${ }^{8}$. Les 73 chefs de ménage se partagent 62 patronymes différents. En 1730, la population est tombée à 192 habitants. Dans les registres paroissiaux, Deveau en retrace quelque 180 , constitués en 46 familles. Seulement onze des noms de 1681 subsistent; $30 \%$ des familles se réclament de cinq de ces patronymes. C'est dire que plus des deux tiers des groupes familiaux

\footnotetext{
7 Gérard Bouchard et al., «La distribution des patronymes au Québec: témoins des dynamiques de population», Anthropologie et Sociétés, 9,3 (1985): 197.

8 Jean Deveau, «Le peuplement de Saint-Barthélemy», extrait du Bulletin de la société d'Histoire de la Guadeloupe (Nérac, Imp. J. Owen), 62 p.
} 
se rangent sous la vingtaine de nouveaux patronymes apparus depuis 1681. Vu la faible homonymie de départ, la réduction de la population devait s'accompagner d'un recul de la diversité. Moins attendue, la substitution de nouveaux noms aux anciens montre bien que les repeuplements successifs n'ont pas fait seulement appel aux habitants déplacés.

Dès 1730 , six patronymes suffisent pour réunir $50 \%$ des familles et tous les six comptent encore aujourd'hui parmi les dix plus fréquents. Quelques décennies plus tard, en 1787, on regroupe les trois quarts des chefs de ménage sous 9 des 10 noms les plus représentés actuellement à Saint-Barthélemy. Seul l'ordre d'importance varie un peu dans le temps (tableau 2). Un observateur de l'époque, le baron de Clugny, écrivait en 1784: «Il n'y a dans l'Isle que 5 ou 6 familles différentes; normands d'origine, ils ont beaucoup multiplié et se marient toujours sans sortir de leur isle. ${ }^{9}$ Ces propos sont visiblement exagérés, mais le stock patronymique s'est néanmoins appauvri entre 1730 et 1787: une quinzaine de patronymes ont disparu; une dizaine d'autres, presque tous uniques, les remplacent. Cette évolution met en évidence la fragilité et le caractère éphémère des marges qui ne cessent de fluctuer et laisse supposer que l'endogamie observée par Benoist ${ }^{10}$ pour la période 1861-1961 est plus ancienne d'au moins un siècle.

À l'arrivée de l'administration suédoise, en 1784, il ne fait pas de doute qu'était déjà en place le noyau dont descend la presque totalité de la population st-barte. Le groupe fondateur était constitué. Les facteurs qui ont concouru à sa survivance et à sa consolidation sont encore peu connus. Avant la paix de 1763 (traité de Paris), la conjoncture a été particulièrement défavorable à l'établissement des habitants: massacres, transplantations, fuites et dispersions sont venus tour à tour saper les efforts de colonisation et ponctionner leur tribut de ressources humaines.

À aucune des réimplantations, l'effectif préexistant n'est pleinement reconstitué, mais à chacune, sans doute, structure et composition sont profondément modifiées. Le dénombrement de $1671^{11}$ présente les traits caractéristiques de nombreuses populations pionnières: forte proportion d'adultes et surmasculinité (170 hommes pour 100 femmes). Cependant, en $1687^{12}$, l'équilibre des sexes semble atteint et la fraction des jeunes a crû sensiblement. Les chiffres de population et les structures de 1731 et $1732^{13}$ montrent qu'on est alors de nouveau en situation

\footnotetext{
9 Cité par Jean Deveau, loc. cit., 20-21.

10 Jean Benoist, «Saint-Barthélemy: Physical Anthropology of an Isolate», American Journal of Physical Anthropology, 22 (1964): 473-487.

${ }_{11}$ Archives nationales de France, Section Outre-Mer (ci-après AN.SOM), G ${ }^{1} 498$, no 54.

12 AN.SOM, G 1498 , no 101.

13 AN.SOM, G1498, nos 110 et 111.
} 
TABLEAU 2

Évolution des patronymes à Saint-Barthélemy, 1681-1979

\begin{tabular}{|c|c|c|c|c|c|c|c|c|c|}
\hline \multirow{2}{*}{$\begin{array}{l}\text { Rolle des } \\
\text { hab. 1681* } \\
\text { Nom }\end{array}$} & \multirow[b]{2}{*}{ Fréq. } & \multicolumn{2}{|l|}{$\begin{array}{l}\text { État civil } \\
1730\end{array}$} & \multicolumn{2}{|c|}{$\begin{array}{l}\text { Recensement } \\
1787\end{array}$} & \multicolumn{2}{|c|}{$\begin{array}{l}\text { Recensement } \\
1846\end{array}$} & \multicolumn{2}{|c|}{$\begin{array}{l}\text { Enq. INSERM } \\
1978-79\end{array}$} \\
\hline & & Nom & Fréq. & Nom & Fréq. & Nom & Fréq. & Nom & Fréq. \\
\hline Aubin & ,01 & Questel & ,11 & Gréaux & ,14 & Laplace & , 15 & Gréaux & 21 \\
\hline Bernier & ,01 & Gréaux & ,09 & Questel & ,13 & Lédée &, 12 & Lédée &, 12 \\
\hline Gréaux & ,01 & Bernier & ,09 & Laplace & , 12 & Gréaux & , 12 & Brin & ,09 \\
\hline Mutrel & ,01 & Aubin & ,07 & Bernier & ,08 & Questel & ,09 & Magras & ,08 \\
\hline \multirow[t]{11}{*}{ Vittet } & ,01 & Laplace & ,07 & Brin & ,08 & Magras & ,08 & Laplace & ,07 \\
\hline & & Lédée & ,07 & Lédée & ,08 & Berry & ,06 & Questel & ,07 \\
\hline & & Vittet & ,04 & Aubin & ,05 & Aubin & ,05 & Aubin & ,06 \\
\hline & & Berry & ,02 & Berry & ,04 & Bernier & ,05 & Blanchard & ,04 \\
\hline & & Brin & ,02 & Magras & ,04 & Brin & ,04 & Berry & ,03 \\
\hline & & Gumbs & ,02 & Danet & ,03 & Danet & ,04 & Bernier & ,02 \\
\hline & & Magras & ,02 & Mayer & ,03 & Duzant & ,04 & Gumbs & ,02 \\
\hline & & Mayer & ,02 & $\mathrm{Coq}$ & ,02 & $\ldots$ & & Turbé & ,02 \\
\hline & & Mutrel & ,02 & Mutrel &, 01 & Turbé & ,01 & Danet & ,01 \\
\hline & & & & Vittet & ,01 & Blanchard &, 01 & & \\
\hline & & Lecoq & & $\begin{array}{l}\ldots \\
\text { Mutrel }\end{array}$ & & Mayer & & Duzant & \\
\hline NP & 62 & & 29 & & 21 & & 36 & & \\
\hline $\mathrm{NF}$ & 73 & & 46 & & 52 & & 215 & & \\
\hline $\mathrm{NH}$ & & & & & & & & & 475 \\
\hline
\end{tabular}

* En 1681, il s'agit des patronymes qui ont persisté et non des plus fréquents. Les patronymes qui apparaissent au cours de la période qui précède sont en italique.

$\mathrm{NP}=$ Nombre de patronymes

$\mathrm{NF}=$ Nombre de familles

$\mathrm{NH}=$ Nombre d'hommes de 18 ans et plus.

Sources: 1681 et 1730: Deveau op. cit., 24-29.

1787: Riksarkivet, Stockholm, Fonds Saint-Barthélemy, XXVIII, 22 et 25.

1846: EDYPH

1978-1979: Institut national de santé et de recherche médicale (France), Unité 155, Structure génétique et pathologie héréditaire de l'isolat de Saint-Barthélemy, enquête médicale, 1978-1979.

de démarrage. Très troublée, la période de 1744 à 1763 va encore trancher dans le vif. Voici comment, en 1763, le procureur général Coquille présente la situation:

L'isle de Saint-Barthélemy est petite et abandonnée. Pendant la guerre de 44, elle fut ruinée par les Anglais qui prirent le peu de Nègres qu'avaient les habitants, et les obligèrent à se réfugier à la Guadeloupe et à la Martinique avec leurs femmes et enfants où ils ont été bien reçus et secourus. Après la paix de 48 , quelques familles y sont retournées, mais sans établissement, et à la guerre de 56, 
elles s'en sont absolument retirées après avoir été pillées par les corsaires. $^{14}$

Le Sieur Descoudrelles, nommé en 1763 gouverneur de SaintMartin et de Saint-Barthélemy, avait entrepris de repeupler ces îles notamment en y ramenant les anciens habitants: «(...) il ne me sera pas difficile d'y attirer ce qui peut rester de familles qui y étaient au commencement de la guerre...» ${ }^{15}$ La persistance de nombreux patronymes de 1730 et les propos du baron de Clugny sur l'homogénéité de la population amènent à postuler que les retours plus tardifs sont venus renforcer des groupes familiaux déjà bien représentés dans l'île et que l'immigration a procédé par réseaux de parenté. On se rappellera que c'est de Saint-Christophe que les Français ont colonisé de nombreuses autres îles dont Saint-Barthélemy, la Guadeloupe, la Martinique... De réelles affinités entre les colons de ces îles ont pu, non seulement entraîner l'établissement hors Saint-Barthélemy d'habitants déplacés, mais aussi l'installation, après 1713 , d'une partie au moins des familles nouvelles à Saint-Barthélemy.

Le temps devait également jouer un rôle certain. D'une part, il suffit d'une génération pour que des familles s'éteignent. D'autre part, l'avance de quelques générations dont bénéficient les familles entrées tôt dans l'île leur confêre un substantiel avantage démographique. Ainsi, en est-il des trois familles pionnières, Gréaux, Aubin et Bernier, recensées en 1681 et de trois autres, Questel, Laplace et Lédée, arrivées entre 1681 et $1687^{16}$, dont les noms ont subsisté. Environ $55 \%$ des hommes de 18 ans et plus portent aujourd'hui ces noms (tableau 2). La probable sélection de familles apparentées dans les vagues de peuplement postérieures au traité d'Utrecht a pu, de son côté, multiplier l'effet du temps, tant pour les familles pionnières que pour d'autres arrivées relativement tôt.

\section{RÉSEAUX FAMILIAUX ET DÉCOUPAGE GÉOGRAPHIQUE}

On peut s'attendre, compte tenu du mode de repeuplement de l'île, à ce que les réseaux d'apparentement aient influencé le modèle d'appropriation des terres. Si le commandant de l'île et son état-major ont vu à contrôler l'établissement des colons, rien n'indique que la procédure adoptée ait été rigoureuse et contraignante. Il semble que, dès sa mise en place, l'administration suédoise ait senti la nécessité d'enregistrer les droits de propriété des habitants de l'île ${ }^{17}$.

\footnotetext{
14 Cité par Jean Deveau, loc. cit., 17.

15 Cité par Jean Deveau, loc. cit., 19.

16 AN.SOM, G 1498 , no 107.

Archives nationales de France, Fonds suédois de Saint-Barthélemy (ci-après AN, FSSB),
} 299 D. 
Une liste des propriétaires ${ }^{18}$, dressée vers 1790 , permet de voir que les «quelques familles» dont parlait de Clugny monopolisent, chacune, des portions bien déterminées de l'île (figure 2). Très peu d'entre elles sont présentes tant $\mathrm{Au}$ Vent que Sous le Vent et jamais en proportions égales. Un même patronyme apparaît généralement dans au plus deux ou trois quartiers limitrophes d'une même partie de l'île. La multiplication, dans un voisinage donné, de parcelles ou d'habitations dont les propriétaires sont homonymes, autorise à évoquer au moins deux pratiques: le regroupement de familles affines dans le processus de repeuplement et l'établissement de plusieurs héritiers autour de l'habitation paternelle ou à même ce patrimoine. Le Suédois Norderling, juge à la cour de justice de Gustavia, déplorait, dans son rapport de 1788, que cette merveilleuse contrée soit aux deux tiers inutilisée ${ }^{19}$. On peut en conclure qu'il y avait, au début de la période suédoise, encore des possibilités d'établissement pour les jeunes et de regroupement pour les familles affines.

Le recensement de 1846 et l'enquête récente de l'INSERM ${ }^{20}$ (19781979) reproduisent à peu de chose près la concentration géographique des patronymes observée à la fin du XVIIIe siècle (tableau 3). On note toutefois une plus large diffusion de certains noms et quelques modifications de leur distribution par quartier. La persistance de la répartition géographique des patronymes mérite d'être considérée à la lumière des valeurs très élevées de l'endogamie de paroisse calculées par Benoist ${ }^{21}$, de la faiblesse de la migration interne observée par Jacynthe Lavoie ${ }^{22}$ et de l'influence de la proximité résidentielle sur l'endogamie de quartier constatée par A. V. James ${ }^{23}$.

\section{LES MARIAGES EXOGAMES}

Pour repeupler l'île, l'opération Descoudrelles faisait appel aux réseaux proprement antillais. Deveau signale un autre apport de population, soit l'arrivée de célibataires venant d'Europe. Une telle immigration, constituée d'individus isolés, n'est généralement que partiellement décelée. On la repère, par exemple, au mariage, au décès ou à un recensement si le lieu d'origine du migrant est mentionné. C'est dire

\footnotetext{
18 Riksarkivet, Stockholm, Collection Saint-Barthélemy, XXVIII, 21.

19 Ernst Ekman, «A Swedish Career in the Tropics: Johan Norderling (1760-1828)», Swedish Pioneer Historical Review, 15,1 (1964): 8.

${ }_{20}$ France, Institut national de santé et de recherche médicale, Unité 155 , «Structure génétique et pathologique héréditaire de l'isolat de Saint-Barthélemy». (1966): 15

Jean Benoist, «Du social au biologique: étude de quelques interactions», L'Homme, 6,1

22 Jacynthe Lavoie, Mobilité interne de la population rurale de l' île Saint-Barthélemy dans les Antilles, de 1854 à 1863. Mémoire de maîtrise (démographie), Université de Montréal, 1986, $141 \mathrm{p}$. Alice V. James, Marriage Patterns in Saint-Barthélemy, French
doctorat (anthropologie), The Pennsylvania State University, 1983, 147 p.
} 
FIGURE 2

Île Saint-Barthélemy, principales divisions et situations dans l'arc des petites Antilles

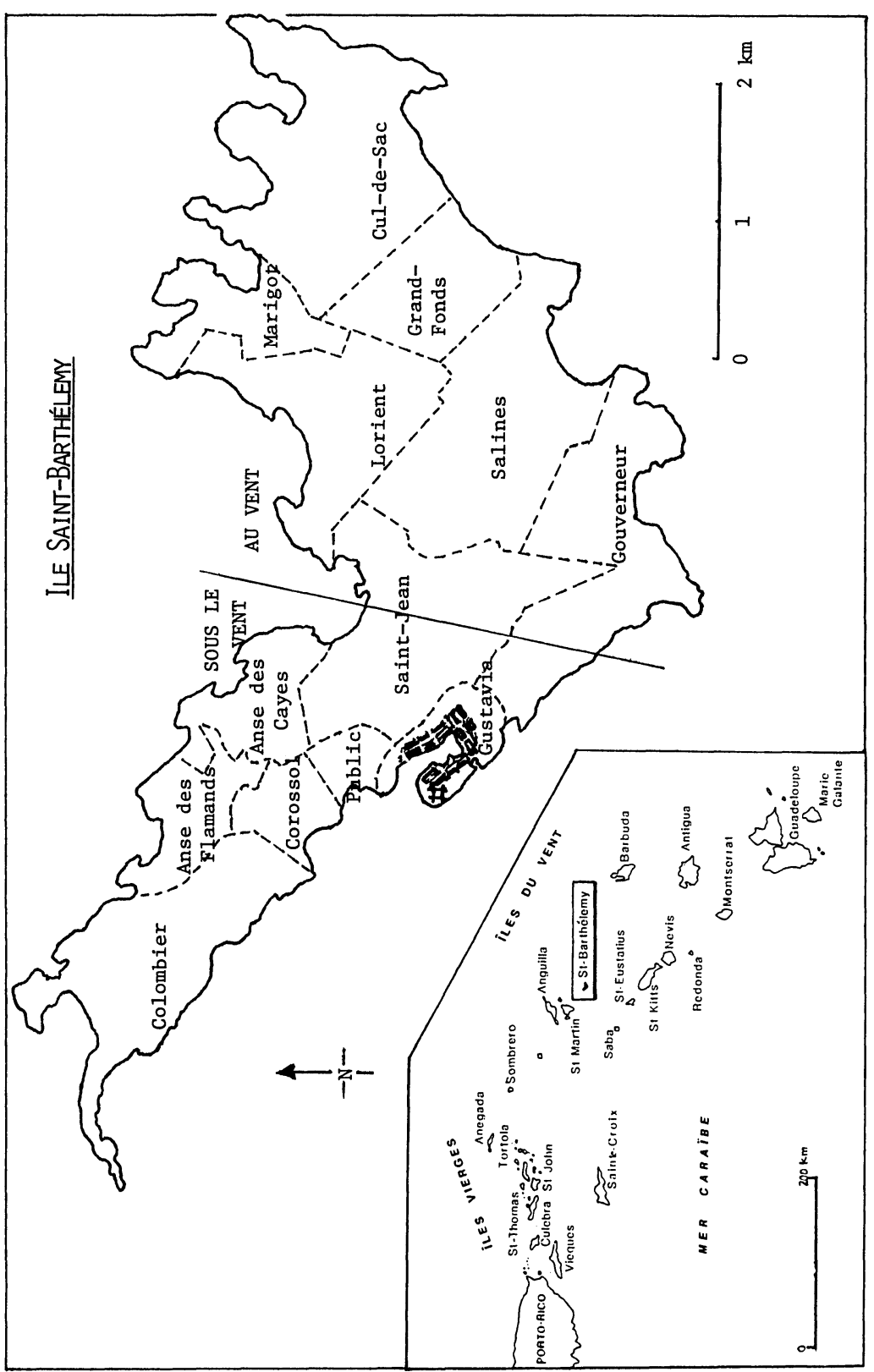


TABLEAU 3

Distribution géographique des principaux patronymes, Saint-Barthélemy, vers 1790 et 1846

\begin{tabular}{|c|c|c|c|c|}
\hline \multirow[t]{2}{*}{ Patronyme } & \multicolumn{2}{|c|}{$\begin{array}{l}\text { Vers } 1790 \\
\text { Forte concentration }\end{array}$} & \multicolumn{2}{|c|}{$\begin{array}{l}1846 \\
\text { Forte concentration }\end{array}$} \\
\hline & Partie & Quartiers & Partie & Quartiers \\
\hline Laplace & $\mathrm{Au}$ Vent & $\begin{array}{l}\text { Lorient, Salines, } \\
\text { Marigot }\end{array}$ & $\mathrm{Au}$ Vent & $\begin{array}{l}\text { Salines, Saint-Jean, } \\
\text { A. des Cailles }\end{array}$ \\
\hline Gréaux & Sous le Vent & $\begin{array}{l}\text { Corossol, Colombier, } \\
\text { Flamant }\end{array}$ & Sous le Vent & $\begin{array}{l}\text { Flamant, A. des } \\
\text { Cailles, Saint-Jean }\end{array}$ \\
\hline Lédée & $\mathrm{Au}$ Vent & $\begin{array}{l}\text { Lorient, Grand Fond, } \\
\text { Salines }\end{array}$ & $\mathrm{Au}$ Vent & Grand Fond, Flamant \\
\hline Questel & Sous le Vent & Colombier, Public & Sous le Vent & $\begin{array}{l}\text { Colombier, Saint-Jean, } \\
\text { Salines }\end{array}$ \\
\hline Magras & Sous le Vent & Colombier, Flamant & Sous le Vent & Colombier, Public \\
\hline Berry & Au Vent & Lorient, Grand Fond & Au Vent & $\begin{array}{l}\text { Grand Fond, Lorient, } \\
\text { Salines }\end{array}$ \\
\hline Aubin & Au Vent & Grand Fond, Marigot & Au Vent & $\begin{array}{l}\text { Grand Fond, Saint-Jean, } \\
\text { Vittet }\end{array}$ \\
\hline Bernier & Au Vent & Saint-Jean, Cul de Sac & $\mathrm{Au}$ Vent & Marigot, Corossol \\
\hline Brin & $\mathrm{Au}$ Vent & $\begin{array}{l}\text { Saint-Jean, Grand Fond, } \\
\text { Salines }\end{array}$ & Au Vent & Grand Fond, Salines \\
\hline Danet & Sous le Vent & Flamant & Sous le Vent & Colombier \\
\hline
\end{tabular}

Sources: vers 1790: Riksarkivet, Stockholm, Fonds Saint-Barthélemy, XXVIII, 21. 1846: EDYPH.

que la probabilité pour un migrant d'être connu par des sources historiques est d'autant plus grande qu'il se sera intégré à la communauté.

Examinant les mariages de la période 1724-1833, Deveau ${ }^{24}$ relève 48 mariages unissant un homme né en Europe, presque toujours en France, et une fille de l'île. Pourtant, peu de noms encore fréquents à Saint-Barthélemy perpétuent le souvenir de ces immigrants français. Citons néanmoins Pierre Danet, marié en 1752, de Nantes; JacquesAndré Blanchard, marié en 1795, de Lille; Joseph Turbé, marié en 1799, aussi de Nantes.

Deveau mentionne également des échanges matrimoniaux entre les habitants de Saint-Barthélemy et ceux de Saint-Christophe, de SaintVincent, de la Martinique, de Curaçao, de Saint-Eustache, de SainteCroix et de Saint-Thomas. Il précise que ces unions intéressent le plus

\footnotetext{
24 Jean Deveau, loc. cit., 30-33.
} 
souvent les mêmes familles ${ }^{25}$. Une telle pratique pourrait traduire la persistance d'affinités solidement établies dans l'île-mère commune: Saint-Christophe. Les nombreuses dispersions de la population st-barte ont également pu fournir aux déportés l'occasion de rafraîchir ou de tisser des liens dans les communautés d'accueil; certains se sont sans doute même établis dans l'île qui les a accueillis. Plusieurs des mariages entre jeunes gens de Saint-Barthélemy et d'autres îles, bien qu'exogames à strictement parler, peuvent être considérés comme une des voies par lesquelles des lignées se sont reconstituées dans l'île après 1763 ou y ont implanté leur réseau.

Même restreinte, l'exogamie aurait pu amener une diversification marquée de la population. L'enrichissement du stock des patronymes dont témoigne le recensement de 1846 s'explique en bonne partie par l'établissement, au moins provisoire, d'étrangers mariés dans l'île. Nous verrons que cet acquis n'a pas davantage persisté que le développement urbain suscité par la Suède.

\section{L'IMPORTANT MAIS ÉPHÉMÈRE GROUPE URBAIN}

Très tôt après sa reconstitution par les soins du Sieur Descoudrelles, la communauté st-barte, passant sous la domination suédoise, est appelée à côtoyer une population étrangère. En effet, un quart de siècle après la cession à la Suède, les quelque 900 blancs d'origine française partagent le milieu avec près de 4000 étrangers (tableau 1). Cependant, les nouveaux venus, contenus dans les limites étroites de la ville de Gustavia, vivent en marge de la communauté rurale française. Les occasions de contact sont d'autant plus rares que la campagne est essentiellement française et catholique et la ville, cosmopolite et multiconfessionnelle.

The Report of St-Bartholomew ${ }^{26}$ ne laisse planer aucun doute sur la coupure entre la ville et la campagne. Publié entre 1804 et 1819 , cet hebdomadaire, rédigé en anglais, rend compte des activités urbaines, fait état d'événements à caractère international, diffuse des directives administratives, mais garde un silence obstiné sur la campagne et ses habitants.

Les recensements nominatifs de la campagne et de la ville (1787 et 1835-1872) témoignent également du clivage rural/urbain. À la campagne, on ne retrouve, à part quelques étrangers très clairsemés, que les habitants d'origine française aux noms bien connus. Parallèlement, à Gustavia, rares sont les patronymes apparus dans l'île avant 1784 .

\footnotetext{
25 Ibid., 30.

26 Kungliga Biblisteket, Stockholm.
} 
À la flambée urbaine succède, dès le lustre 1815-1819, un marasme persistant. Le temps et la bonne fortune auront manqué pour qu'il y ait eu, même timidement, une pénétration durable d'un milieu par l'autre. Un peu avant 1820 , ville et campagne avaient commencé à déverser vers l'extérieur leurs excédents démographiques respectifs.

\section{L'ÉROSION MIGRATOIRE}

C'est donc relativement tôt, sous la domination suédoise, que s'amorce une lente et efficace érosion tant des gains dus à l'immigration que de l'excédent des naissances sur les décès. Alors que la ville s'engage sur la voie d'un déclin économique et démographique sans appel, la saturation du terroir freine la croissance démographique du milieu rural. Celui-ci commence même à subir des pertes vers 1830 (figure 1). L'émigration qui ne laisse pas d'inquiéter l'administration aurait adopté un déroulement inverse de celui qu'a suivi le repeuplement. La première vague emporte plusieurs des familles fondées par des célibataires européens qui ont pris femme dans l'île au tournant du XIXe siècle: Babin, Bercier, Bossel, Cousin, Curet, Faltaboule, Jambrun, Masson, Robinet, etc. On en retrouve le plus grand nombre à Porto Rico et les autres surtout à la Martinique, à Saint-Thomas, à Saint-Martin et à la Guadeloupe. Le relais est pris par des familles que l'exode, entre autres facteurs, a amenées, sinon à l'extinction, du moins à un très faible poids relatif dans la population de l'île. Et, cyclones, sécheresses, incendie ajoutant leurs effets aux conséquences de l'excessif morcellement des terres signalé dès 1820 , le mouvement d'émigration s'est de plus en plus nourri à même les lignées les mieux établies dans l'île.

La piètre situation économique de Saint-Barthélemy rend la population perméable aux sollicitations du gouverneur de Porto-Rico qui recrute des colons catholiques et à la demande de main-d'oeuvre venant principalement de Saint-Thomas (Îles Vierges) et des colonies privées d'esclaves (l'abolition de l'esclavage a eu lieu en 1834 dans les colonies anglaises). Le gouverneur Haasum écrivait, à la fin des années 1830:

L'émigration de cette île qui depuis la décadence du commerce se portait principalement sur Saint-Thomas et Porto Rico a dernièrement reçu une nouvelle direction. Le manque d'ouvriers dans la colonie de Demerara où il paraît qu'on obtient une haute prime de tant par tête pour chacun de ceux qui y sont importés, a donné lieu à une espèce de traite (...) 70 à 80 personnes, hommes et femmes (...) sont partis pour Demerara (26 septembre 1836).

La campagne a eu à supporter de grandes souffrances et privations en conséquence de la grande sécheresse qui s'est prolongée jusqu'à présent $(. .$.$) . La misère a chassé vingt-sept de ces gens de leurs$ foyers et ils sont partis pour Demerara s'étant engagés moyennant quelques avances à un agent qui est venu ici (...) (20 juillet 1837). 
Des cent cinquante campagnards qui émigrèrent pour la colonie de Demerara, 33 seulement sont revenus dernièrement dans un très mauvais état de santé; tous les autres ayant péris par suite du changement de climat (25 juillet 1938). ${ }^{27}$

Le caractère sélectif de l'émigration devait renforcer la prépondérance des vieilles familles. Ainsi, les immigrants célibataires européens n'ont que peu modifié le noyau à peine formé. Les premiers, ils ont fait les frais de l'émigration devenue nécessaire peu de temps après qu'ils eussent pénétré au coeur de la communauté st-barte par des unions avec des familles de souche.

Lorsqu'elle touchait les vieilles familles st-bartes, l'émigration pouvait contribuer à la contraction du noyau. On peut penser en effet que ceux que la communauté avait le moins intégrés ou qui s'y sentaient le moins à l'aise ont été les plus susceptibles de quitter l'île. L'intrication des lignées qui persistent devient à ce point poussée que des jeunes gens, exclus du cercle des mariables par la multiplication des apparentements, sont contraints au célibat ou à l'émigration ${ }^{28}$.

Quant à la population de couleur, à Gustavia, elle se raréfie rapidement, tout comme le groupe de souche européenne, à partir de 1820 environ. Après l'abolition de l'esclavage (1846-1847), les ruraux de couleur ne resteront pas davantage. On ne sait ni comment ils sont partis ni quelles solidarités extérieures ils ont pu mettre en oeuvre: presque tous étaient nés à Saint-Barthélemy. Après l'abolition de l'esclavage, il y a concurremment redistribution géographique du groupe qui se concentre de plus en plus vers le centre de l'île et fléchissement de sa croissance $^{29}$. Lorsqu'on suit les individus d'un recensement à l'autre, il apparaît que les gens de couleur sont proportionnellement plus nombreux que les blancs à sortir d'observation ${ }^{30}$, donc selon toute probabilité à émigrer. Il serait difficile d'interpréter autrement l'extinction du groupe qui s'est consommée sans qu'il soit possible, avec la documentation existante, d'en suivre le déroulement dans le temps et dans l'espace.

Ainsi, le vieux noyau de population blanche et française a pu progressivement occuper l'enclave urbaine laissée vacante par les Suédois et l'espace démographique libéré par les gens de couleur. On est amené à penser que l'émigration a également permis de relâcher les tensions sociales et de renforcer l'emprise du noyau.

$27 \quad$ AN, FSSB, 50 Mi 125.

28 Paul W. Leslie, Bennettt Dyke et Warren T. Morrill, «Celibacy, Emigration and the Genetic Structure in Small Population», Human Biology, 52,1 (1980): 115-130.

29 Denis Gonthier, Abolition de l' esclavage et transformation des ménages de la population rurale de couleur, île antillaise de Saint-Barthélemy, de 1840 à 1857. Mémoire de maîtrise (démographie), Université de Montréal, 1987, 127 p.

30 Jacynthe Lavoie, op. cit. 
Comme telle, l'ampleur de l'exode des ruraux d'origine française n'est pas connue. Le mouvement a été suffisant pour donner naissance à deux communautés st-bartes dans l'île Saint-Thomas (Îles Vierges): North Side et Carénage et pour nourrir des courants migratoires moins visibles vers les États-Unis et la France en particulier.

Une simple simulation, fondée sur l'accroissement naturel, permet d'appréhender l'importance du déficit démographique dû à l'émigration. Les propos tenus par le baron de Clugny en 1784 inclinent à penser que la taille des familles st-bartes n'était pas très éloignée de celle des familles canadiennes-françaises du XVIIle siècle: "A travers des halliers épais on arrive par des sentiers détournés à une mauvaise case où reste un homme et une femme avec 7 à 8 enfants qui le jour travaillent comme des nègres et la nuit se couchent pêle et mêle. ${ }^{31}$

Ce témoignage d'une forte fécondité est corroboré par des mesures indirectes effectuées à partir des recensements du milieu du XIXe siècle $^{32}$. Une mortalité généralement élevée et marquée par des pointes de surmortalité a ralenti le rythme de l'accroissement naturel, mais c'est surtout l'émigration qui a contenu l'expansion du nombre des habitants. La croissance annuelle moyenne des blancs à la campagne se maintient à $2,5 \%$ entre 1784 et 1812 , période de faible immigration pour ce groupe, mais tombe à $0,4 \%$ entre 1812 et 1840 , alors que l'émigration sévit.

Avec un accroissement naturel moyen de $1,25 \%$ par an, soit seulement la moitié de celui observé chez les Canadiens français jusqu'à la fin du XIXe siècle ${ }^{33}$, les quelque 500 habitants d'origine française de 1787 auraient engendré une descendance conduisant à un effectif de 6000 individus vers 1980. En haussant l'accroissement naturel à 1,5\% par an, on obtiendrait près de 10000 habitants à la même date. Cette simulation qui n'a rien de déraisonnable sur la longue période permet d'entrevoir l'ampleur du rôle joué par les sorties au plan purement démographique.

Soupape contrôlant les pressions démographique et sociale à un niveau très fin, l'émigration externe a aussi sans doute contribué au maintien du très ancien découpage de l'île en deux parties et une quinzaine de quartiers et à la persistance du cloisonnement des populations circonscrites (figure 2). Peut-on autrement expliquer la diversité linguistique et biologique que rapportent Benoist et Lefebvre: ${ }^{34}$

31 Cité par Jean Deveau, loc. cit., 21.

32 Yolande Lavoie et al., «Structure et mouvement de la population blanche et rurale de l'île de Saint-Barthélemy, 1846-1857», Culture, 4,1 (1984): 55.

33 Jacques Henripin et Yves Péron, «La transition démographique de la province de Québec», Hubert Charbonneau, La population du Québec: études rétrospectives (Montréal, Les Éditions du Boréal Express, 1973), 43.

34 Jean Benoist et Gilles Lefebvre, «Organisation sociale, évolution biologique et diversité linguistique à Saint-Barthélemy», in L'archipel inachevé par J. Benoist, (Montréal, Presses de l'Université de Montréal, 1972): 101-103. 
La coupure majeure de l'île en deux entités sociales, le Vent et Sous le Vent, correspond également à une coupure majeure du point de vue linguistique: Sous le Vent parle principalement le français et ses variantes régionales (...); le Vent a au contraire subi une créolisation poussée (...). La plus grande homogénéité de la paroisse de Lorient (le Vent) (...) a pour parallèle une plus grande homogénéité linguistique (...). Inversement les quartiers de la paroisse de Gustavia (...) abritent plusieurs idiomes.

(...) au moment d'interpréter les résultats des mensurations et des groupes sanguins, il est devenu évident que l'île de Saint-Barthélemy ne pouvait être traitée comme un ensemble unitaire (...). Du point de vue biologique, on ne se trouvait pas en présence d'une population, mais de plusieurs populations qui n'avaient entre elles que des échanges restreints (...)

\section{CONCLUSION}

Le tableau qu'on a pu tirer d'une documentation lacunaire et disparate laisse de nombreux points dans l'ombre. Il bénéficiera des raffinements que lui ajouteront les recherches en cours auxquelles il fournit un contexte éclairant et des éléments d'interprétation.

À quatre reprises au moins, au cours du premier siècle de colonisation, la population de l'île est soit réduite à néant, soit très largement amputée. Le dernier peuplement, postérieur au traité de Paris, est avant tout un remembrement: l'île est principalement livrée à d'anciennes familles, formant réseau par apparentement ou affinités diverses. Il a suffi à ces familles de trois décennies pour se réapproprier l'île et y consolider leur emprise. En 1787, elles se distribuent selon un découpage en quartiers sans doute hérité de la période 1713-1747; chaque quartier est dominé par un groupe bien défini de sorte qu'il y a correspondance plus ou moins étroite entre la carte des quartiers et celle des patronymes.

L'expansion du groupe rural français accompagne l'explosion urbaine consécutive à la cession de l'île à la Suède. Pendant cette courte période, la communauté rurale voisine avec une population étrangère sensiblement plus nombreuse. Elle se fait faiblement assimilatrice et se montre réfractaire à l'assimilation à laquelle elle n'est que brièvement exposée. Dès la troisième décennie du XIXe siècle, la population de l'île est soumise à une érosion continue qui emporte d'abord les effectifs urbains et de couleur et laisse dans l'île, dans leur quartier d'origine le plus fréquemment, les éléments irréductibles du «vieux noyau» stbart.

Les recherches en cours visent à inscrire sur cette toile de fond le jeu des alliances, l'effet des différences de fécondité et de mortalité, les rôles sélectif et palliatif des migrations, en relation avec les systèmes qui les contrôlent. Les reconstructions généalogiques, en cours pour le 
XVIIIe et le début du XIXe siècle, montreront infiniment mieux la contribution des familles fondatrices et les échanges entre lignées que les structures patronymiques tronquées de l'apport féminin. Sont également en progrès des analyses de la composition des ménages, des réseaux de voisinage et de leurs transformations, éléments puissamment révélateurs de la dynamique qui sous-tend l'organisation sociale et l'évolution biologique. 